\title{
Effects of surface stability on the morphological transformation of metals and metal oxides as investigated by first- principles calculations
}

\author{
Juan Andrés ${ }^{1}$, Lourdes Gracia ${ }^{1}$, Amanda Fernandes Gouveia ${ }^{2}$, \\ Mateus Meneghetti Ferrer ${ }^{2}$ and Elson Longo ${ }^{3}$ \\ ${ }^{1}$ Department of Analytical and Physical Chemistry, University Jaume I (UJI), Castelló E-12071, Spain \\ ${ }^{2}$ INCTMN-UFSCar, Universidade Federal de São Carlos, PO Box 676, 13565-905 são Carlos, SP, Brazil \\ ${ }^{3}$ INCTMN-UNESP, Universidade Estadual Paulista, PO Box 355, CEP 14801-907 Araraquara, SP, Brazil \\ E-mail: andres@qfa.uji.es
}

Received 30 June 2015, revised 13 August 2015

Accepted for publication 14 August 2015

Published DD MM 2015

\begin{abstract}
Morphology is a key property of materials. Owing to their precise structure and morphology, crystals and nanocrystals provide excellent model systems for joint experimental and theoretical investigations into surface-related properties. Faceted polyhedral crystals and nanocrystals expose well-defined crystallographic planes depending on the synthesis method, which allow for thoughtful investigations into structure-reactivity relationships under practical conditions. This feature article introduces recent work, based on the combined use of experimental findings and first-principles calculations, to provide deeper knowledge of the electronic, structural, and energetic properties controlling the morphology and the transformation mechanisms of different metals and metal oxides: Ag, anatase $\mathrm{TiO}_{2}, \mathrm{BaZrO}_{3}$, and $\alpha-\mathrm{Ag}_{2} \mathrm{WO}_{4}$. According to the Wulff theorem, the equilibrium shapes of these systems are obtained from the values of their respective surface energies. These investigations are useful to gain further understanding of how to achieve morphological control of complex three-dimensional crystals by tuning the ratio of the surface energy values of the different facets. This strategy allows the prediction of possible morphologies for a crystal and/or nanocrystal by controlling the relative values of surface energies.
\end{abstract}

Keywords: morphology, Wulff theorem, surface energy

SQ1 (Some figures may appear in colour only in the online journal)

\section{Introduction}

The ability to prepare nano- and microstructures with a welldefined morphology and excellent monodispersity at the nano- and bulk scale is an essential requirement for materials applications [1, 2]. It is generally accepted that the morphology, dimensionality, and crystal structure of the materials all play important roles in the electronic, optical, magnetic, catalytic, chemical, and other physical properties [3, 4]. Crystal morphology is governed by anisotropic surface properties, i.e., the presence of face-specific molecular arrangements, and is a critical determinant of the physical and chemical properties of crystalline materials. These molecular arrangements stem from the crystal structure and possess a symmetry that allows a group of faces, known as a family, to share identical arrangements. The anisotropic surface properties control the surface energies, growth rates, dissolution rates, wettability, cohesion, adhesion, etc. Because of the presence of surface energy anisotropy, unstable surfaces try to stabilize through the creation of low-energy surfaces; this is commonly called faceting [5]. The control of crystal morphology is a complex and difficult process, which depends 
both on the crystal internal structures and external growth conditions such as the synthesis process, the solvents used, and the process additives. In this context, Xue et al have developed a chemical bonding theory of single crystal growth $[6,7]$. Very recently [8], these authors have reported the morphology engineering of electrode materials that can increase the performance of electrochemical energy storage devices such as $\mathrm{Cu}_{2} \mathrm{O}$ - and $\mathrm{MnO}_{2}$-based systems.

The relative growth rate of crystal faces, which depends on environmental conditions, evokes transformations in crystal morphology because of the appearance and/or disappearance of faces. These transformations are due to the geometric constraints imposed by the crystal structure and are associated with the relative surface energy values of each face. The observed crystal shapes are often interpreted in terms of Wulff construction [9, 10] offers a simple method to determine the equilibrium crystal shape, and different numerical implementations of this procedure are available in the literature [11-13]. Such a description is appropriate at the thermodynamic equilibrium.

This study reports results from theoretical and computational chemistry, in particular, the combination of firstprinciples calculations with an algorithm based on the Wulff construction, to find suitable morphological structure candidates. This perspective presents a comprehensive framework to rationalize crystal morphology and morphology evolution in crystalline materials, and summarizes our research efforts, which target the determination of the morphology in a set of materials ranging from metals to both simple and complex metal oxides: $\mathrm{Ag}$, anatase $\mathrm{TiO}_{2}, \mathrm{BaZrO}_{3}$, and $\alpha-\mathrm{Ag}_{2} \mathrm{WO}_{4}$. The following discussion is divided into three sections. The first section focuses on the state-of-the-art developments in this research field. The theoretical procedure and computational method are presented in section two. The third section presents the results and a process of how to create a mapping of all shape crystal possibilities to evaluate the methods to simulate suitable morphological structural candidates in order to obtain the experimental morphology of the as-synthesized samples.

\subsection{State-of-the-art developments}

For a crystalline material, different surfaces may have different geometric and electronic structures, and exhibit different physical and chemical properties. Accordingly, their morphology is dependent on the surface energies of the different exposed surfaces, and one may expect to tune the properties of materials by simply controlling the morphology of the crystalline materials. At the nanoscale, the ability to control the size and shape of nanocrystals (NCs) in order to tune functional properties is an important goal. The precise fabrication of NCs with specific shapes at the nanoscale level is crucial for enhancing performance in many applications [14-16]. The techniques for nanomaterial synthesis are the pillars of nanoscience and nanotechnology [17, 18]. Nanomaterial syntheses can be fine-tuned to tailor the shape of the nanomaterials, which enables the application of nanomaterials in various fields, such as catalysis [19-21] electronics [22], optics [23], and magnetism [24] etc. Hence, developing a methodology for shape-controlled NC growth is a major current research direction.

It is well established that structural changes at surfaces, including atomic relaxation and reconstruction, are a manifestation of the driving force to minimize the surface energy $[25,26]$. Such shape effects result inherently from the atomic arrangement of different exposed crystal facets, and the growth mode of an $\mathrm{NC}$ is determined by the ratio between the growth rates of different facets [27]. Recent studies have shown that the use of capping agents is a typical and efficient strategy to control morphology and size. The functional groups on these capping agents can preferentially adsorb onto the crystal facet and modulate the kinetics of crystal growth [28-30]. Maneuvering the surface energies and growth rates of different facets determines the shape of a NC [19, 31]. Shape control of crystal growth has been reported for various types of oxide materials by modulating the reactive conditions in the liquid phase $[32,33]$. In particular, surface charges and polarization have been discussed in previous reports on the synthesis of nanomaterials with some novel morphologies $[34,35]$. However, the synthesis of crystals with the desired morphologies requires an understanding of the thermodynamic and kinetic mechanisms of the formation of NCs and a framework to guide the selection of environmental conditions.

Even at the nanoscale, fine control over nanoparticle (NP) morphology (surface structure) has also been shown to induce superior catalytic reactivity and selectivity [36, 37]. It is also possible to discover new and potentially useful compositions of matter on the nanoscale, because mixtures of elements that are thermodynamically unstable in the bulk may actually become stable (or metastable) as a result of quantum size-confinement effects [38, 39]. As Pauli said 'God made the bulk; surfaces were invented by the devil'.

Identifying the mechanism of the morphological transformation of both nano- and micro-crystals is crucial to gain better control of the growth of materials because the surface structure can strongly affect the crystal quality and properties. The conventional understanding of the surface atomic structure of a crystal is that facets with a higher percentage of under-coordinated atoms are usually more reactive. Modulation of morphology is a commonly employed strategy to optimize the performance of various crystalline catalysts from noble metals to semiconductors [40-42]. The synthesis of highly active facets has been considered as an important procedure to significantly promote the catalytic activity of noble metals, metal oxides, and semiconductors [43-46]. In particular, the peculiar particle morphological structures of metals and their oxides, such as cube, flower-like, octahedron, and rod, have attracted wide attention in the field of electrochemical device applications [47-49]. The basis of this strategy is that the surface atomic configuration and coordination control the reactivity and can be finely tuned by morphological control [50, 51]. The chemistry of diverse energy facets and the differences in the binding properties of the intermediates and/or products with such facets are additional determining factors in deciding the overall catalytic 
activity of the materials under operating conditions. However, many questions still remain because of the lack of conclusive evidence about the mechanisms that govern crystal facet modulation at the atomic level; as such, there is considerable scope for research. In this strategy, insights from theoretical calculations and experimental evidence can be integrated with each other to develop a simple model that can be used to better understand crystal facet modulation mechanisms at the atomic level.

First-principles investigations have been recognized as effective for studying the morphology and surface properties of a wide variety of crystals and NCs [52-54]. Our group has developed a working methodology, which has been applied to the study of different morphologies in different metal oxides such as $\mathrm{SnO}_{2}$ [55-57], $\mathrm{PbMoO}_{4}$ [58], and $\mathrm{CaWO}_{4}$ [59]. The relative growth rates of crystal faces, which depend on environmental conditions, cause transformations in crystal morphology because of the appearance and/or disappearance of faces. These transformations are due to the geometric constraints imposed by the crystal structure and are associated with the relative values of the surface energy of each face. In this context, Roca et al published some results about our simple way to use the theoretical morphology modulation in $\mathrm{TiO}_{2}$ and $\alpha-\mathrm{Ag}_{2} \mathrm{WO}_{4}[60,61]$ nanocrystals in order to explain the surface energy changes observed in experimental conditions.

The main practical challenges of the present work can be summarized as follows: (i) prediction of different morphologies possible for a crystalline material, (ii) screening of relative values of surface energies to obtain desired morphologies, and (iii) target and control of crystal morphologies.

\section{Theoretical procedures and computational methods}

The task of predicting the morphology of crystals can be partially tackled if interest is limited to general knowledge about trends regarding bulk and surface energies (values and variations). The energetic interplay between surface energy and morphology can be identified. This section briefly presents the definition of surface energy and the Wulff construction, which are the most basic parameters and methods for predicting the equilibrium morphology of a crystal.

From the thermodynamic point of view, the equilibrium shape of a crystal is determined by the free energies of various facets, and can be calculated by the classic Wulff construction that minimizes the total surface free energy at a fixed volume [9]. The Wulff theorem provides a simple relationship between the surface energy, $E_{\text {surf, }}$ of the (hkl) plane and its distance, rhkl, in the normal direction from the center of the crystallite. Later, the thermodynamic stability of the faceted and rounded shapes was revisited by Herring [10]. In the case when the morphology of a $\mathrm{NC}$ is known from microscopic observations, $E_{\text {surf }}$ can be assessed iteratively by using the Wulff construction in the inverse fashion until the resulting shape matches the observed shape [11].
First-principles calculations were carried out using the density functional theory and implemented in the Vienna $a b$ initio simulation package in order to find an ideal structure in vacuum $[62,63]$. The Kohn-Sham equations were solved by means of the Perdew-Burke-Ernzerhof exchange-correlation functional [64] and the electron-ion interaction was described by the projector-augmented-wave pseudo potentials $[62,65]$. The plane-wave expansion was truncated at a cut-off energy of $520 \mathrm{eV}$, and the Brillouin zones were sampled through the Monkhorst-Pack special k-points grids. The calculations were done by considering slabs with thickness of up to $\sim 20 \AA$. They are sufficient to reproduce bulk-like properties at the center of the slabs, to obtain a careful description of the surfaces and to reach convergence on the corresponding energy surface values. A vacuum spacing of $15 \AA$ is introduced in the $z$-direction so that the surfaces will not interact with each other. The positions of all atoms of the slab were allowed to relax, and the conjugated gradient energy minimization method was used to obtain relaxed systems, accomplished by requiring the forces experienced by each atom to be smaller than $0.01 \mathrm{eV} \AA^{-1}$. $E_{\text {surf }}$ is defined as the total energy per repeating cell of the slab $\left(E_{\mathrm{slab}}\right)$ minus the total energy of the perfect crystal per molecular unit $\left(E_{\text {bulk }}\right)$ multiplied by the number of molecular units of the surface $(n)$, divided by the surface area per repeating cell of the two sides of the slab, as following:

$$
E_{\text {surf }}=\frac{E_{\text {slab }}-n E_{\mathrm{bulk}}}{2 A} \text {. }
$$

The total-energy calculations are strictly valid only at zero temperature, and the entropic contribution can be taken into account by adding the computed full phonon density of states [66] or alternatively, in a simplified treatment, by inclusion of the most relevant vibration modes [67]. The crystal habits are principally determined by relative energies of the exposed planes, and entropy terms normally do not affect this ordering [68]. The entropic and $\mathrm{pV}$ contributions to the free energies change slightly throughout the condensedphase species and in this type of calculation the entropic term is neglected [68-73]. Therefore, the difference between the Gibbs free energies can be approximated by the difference between the total energies from first-principles calculations [74-76].

Crystallite shape of any kind of materials can, in turn, be determined using the Wulff theorem and construction [9, 77] taking into accounts the surface energy values, implemented in the SOWOS program [13]. In this paper, this methodology was used for the morphology mapping of $\mathrm{Ag}$, anatase $\mathrm{TiO}_{2}$, $\mathrm{BaZrO}_{3}$, and $\alpha-\mathrm{Ag}_{2} \mathrm{WO}_{4}$ systems.

Several insightful review papers have reported experimental and theoretical calculations on the important aspects that govern crystal shape modulation in semiconductor and metal NPs [78-80]. In addition to the thermodynamic driving forces, kinetics also plays an important role in the determination of the morphology under real growth conditions. In such a case, the approach developed by Chernov can be applied to predict the steady-state shapes of NCs [81], and 


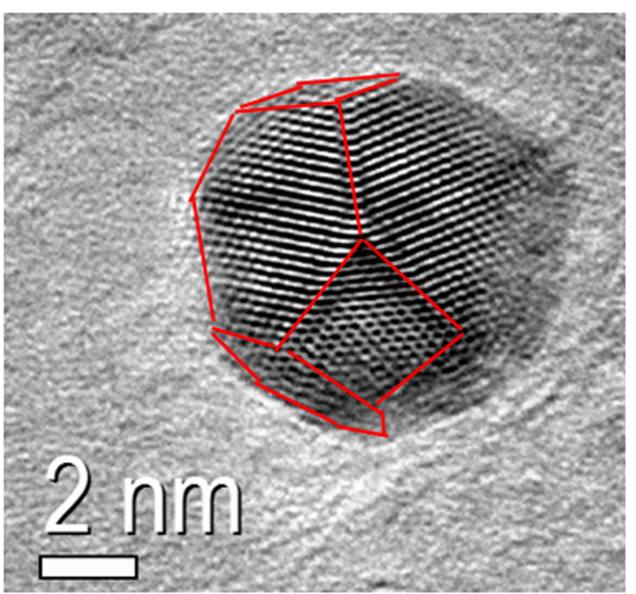

Figure 1. TEM image of $\mathrm{Ag} \mathrm{NC}$ formed under electron beam irradiation.

different authors have developed strategies to calculate the velocity of facet growth $[82,83]$. It was necessary, therefore, to develop a novel strategy to construct polyhedral structures and to especially address such structural issues.

\section{Results and discussion}

\section{1. $\mathrm{Ag}$}

Noble metal NPs with defined structures and novel compositions are of great interest because of their potential applications in a broad range of industrially important processes $[14,84,85]$. Over the past decade, Ag NCs with a myriad of shapes (e.g., spheres, cubes, octahedrons, right bipyramids, bars, plates, rods, and wires) have been synthesized using various methods [86-88].

Recently, our research group reported the unprecedented real-time in situ observation of the growth process of silver metallic $\mathrm{Ag}$ filaments from an unstable $\alpha-\mathrm{Ag}_{2} \mathrm{WO}_{4}$ crystal matrix when the crystal was subjected to electron irradiation from a transmission electron microscope (TEM) [89]. This is a striking result and the mechanism associated with the early events of the nucleation process of $\mathrm{Ag}$ has been studied in detail [90]. The crystal growth mechanism under electron beam irradiation observed in our work is completely free of additional agents, and the in situ TEM enables the visualization of growth of Ag NPs with planar resolution. However, an investigation of the reactions and crystal growth mechanism under electron beam irradiation can be very complicated and demands new studies [91].

An expanded image of the region around the electron driven Ag filament is illustrated in figure 1, where it is possible to identify the morphology of the Ag NC formed during the experiments. In addition, the observed $\mathrm{NCs}$ tend to aggregate into clusters that interact in close proximity, which implies the possible existence of an attractive force among the NCs [92, 93].

Thermodynamic calculations predict that single crystals of face-centered cubic materials such as Pt or Ag will form

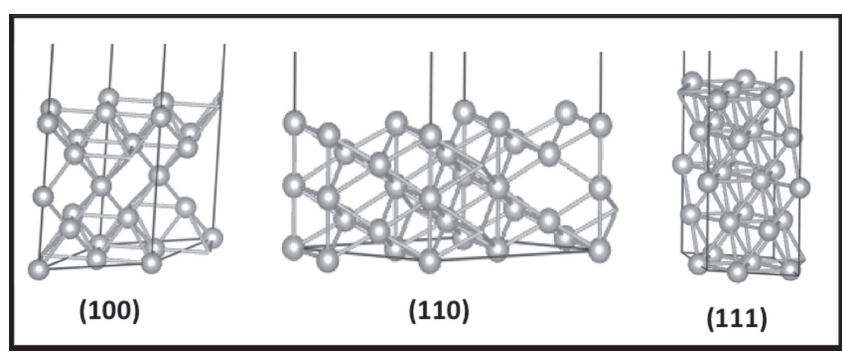

Figure 2. Surface models of (100), (110), and (111) for Ag.

truncated octahedral shapes, with the ratio of their (111) [49], and (100) type surfaces predicted through consideration of the ratio of their two respective surface energies [94]. This ratio can be significantly and controllably modified through the use of surfactants, and it has been demonstrated to have a substantial effect on catalytic selectivity [36]. Particle morphology also dictates the nearest-neighbor coordinations of the exposed surface atoms, broadly affecting the catalytic activity [95].

The morphological characterization of Ag NPs has shown strong correlations between properties such as average particle size, shape, and dispersity with observed and inherently tunable physiochemical behavior [96, 97]. In this context, Barnard et al [98] have presented detailed structure/ property maps, showing that faceted near-spherical shapes and spheres are the predominant morphologies in Ag NPs colloidal samples at low and high temperatures. The production of well-defined NPs with reproducible size and shape distributions remains challenging [99], although different studies report clarification of stability, probability, and population in NP ensembles [100]. The (100), (110), and (111) surfaces were modeled by unreconstructed (truncated bulk) slab models by using a calculated equilibrium geometry and using a $(6 \times 6 \times 1)$ Monkhorst-Pack special k-points grid to ensure geometrical and energetic convergence for the $\mathrm{Ag}$ structures considered. Slab models containing $15 \mathrm{Ag}$ atoms placed in 3 five-atomic layers were selected, with areas of $8.77 \AA^{2}, 12.4 \AA^{2}$, and $7.59 \AA^{2}$ for the (100), (110), and (111) surfaces, respectively. Figure 2 depicts the slab representation for $\mathrm{Ag}$.

An analysis of figure 3 shows that the calculated energy surface values (center of figure 3) generate a morphology very similar to that of synthesized Ag NCs, generated by matching the corresponding TEM image (see figure 1). When the difference in the stability of the facets is reduced, more than one type of facet will appear, and the resulting polyhedron should gradually appear rounder, with truncated edges and corners. When the ratio of the energy surface is varied by increasing the value of the (111) surface more than $22 \%$ because of some perturbation, the resulting morphology is a double truncated octahedron. The same morphology is obtained by decreasing the value of the (110) and (011) surfaces more than $24 \%$ with respect to the computed initial value. On the other hand, a truncated cube or a truncated octahedron can be achieved when the value of the (100) and (001) surfaces is decreased by around $42 \%$ or is increased by around $20 \%$, respectively. 


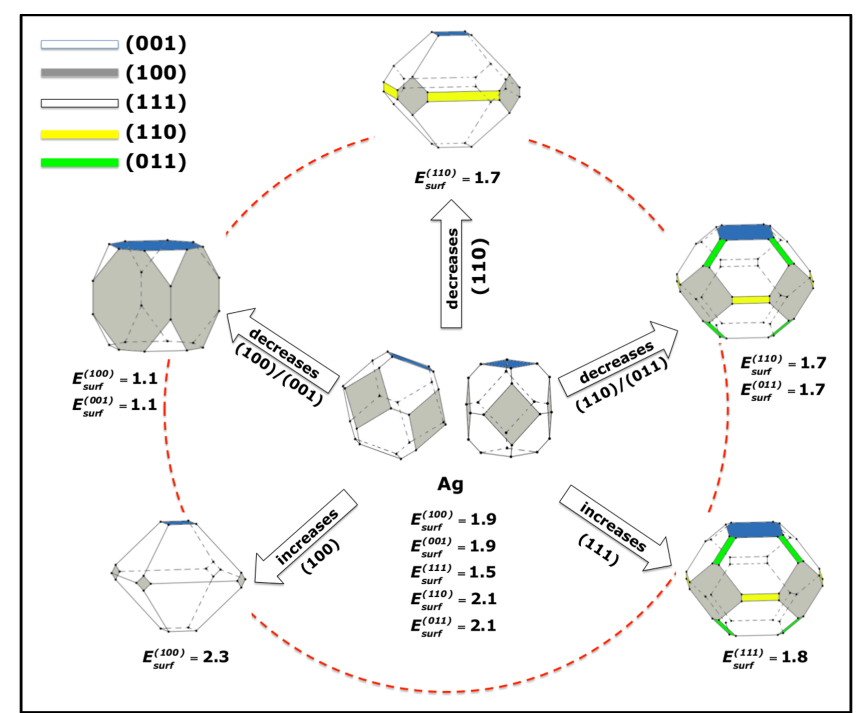

Figure 3. Crystallographic structure and map of morphologies of Ag NC with (100), (001), (111), (110), and (011) crystal planes. Surface energy is in joule per·square meter. Theoretical (experimental) [102] lattice parameter, $a=4.188 \AA$ (4.086 ̊).

In addition, using the different morphologies displayed in figure 3, we can rationalize some experimental results reported very recently by Wang et al [101] such as the formation of $\mathrm{Ag}$ nanocubes and octahedra.

\subsection{Anatase $\mathrm{TiO}_{2}$}

The morphology as well as the crystal-facet-controlled synthesis of titanium dioxide $\left(\mathrm{TiO}_{2}\right)$ has attracted interest because of its scientific and technological applications, including in photocatalysts and self-cleaning agents [103].

$\mathrm{TiO}_{2}$ is a common multifunctional material, which has been used in a wide variety of applications in many fields, such as in ceramics, cosmetics, medicine, food, and catalysts [104]. For $\mathrm{TiO}_{2}$, three polymorphs are stable at atmospheric pressure: the thermodynamically favored rutile phase that can be grown in bulk, and the less common anatase and brookite phases. The polymorphs have significantly different chemical reactivities with anatase and brookite typically being believed to be the most reactive. Hence, many researchers have pursued polymorph-specific syntheses of high-reactivity NCs [105]. Anatase $\mathrm{TiO}_{2}$ was proven to be more active than the other two main $\mathrm{TiO}_{2}$ crystal polymorphs, i.e., rutile and brookite, especially when $\mathrm{TiO}_{2}$ is employed as a catalyst and photocatalyst $[50,106]$.

Under equilibrium conditions, natural and synthetic anatase $\mathrm{TiO}_{2}$ single crystals mainly exist in an octahedral bipyramidal morphology in which a majority of the surface is normally enclosed by energetically favorable (101) facets rather than the more reactive (001) facets [107-109]. Thus, it has long been a great challenge to synthesize anatase $\mathrm{TiO}_{2}$ with a large fraction of exposed (001) facets. However, recently, many studies have reported the synthesis of highenergy anatase surfaces $[42,110,111]$.
Despite intense investigations into the preparation of specific facets, the inherent photoactivities of $\mathrm{TiO}_{2}$ facets are still ambiguous. The (001) facet was initially expected to be the most active because of its high surface energy [42, 112115], whereas later research suggested that the clean (010) facet with $100 \%$ five-coordinate $\mathrm{Ti}$ was more active in photooxidation and photoreduction reactions [116]. In particular, Liu et al reported that the photoreactivity of (010) facets of $\mathrm{TiO}_{2}$ is higher than that of the (101) facets, while the (001) facets showed the lowest photoreactivity [116]. However, more recent research has suggested that different facets may play different roles in photoreaction $[117,118]$.

Based on first-principles calculations, the $E_{\text {surf }}$ values for (101), (001), (110), and (100) facets of anatase $\mathrm{TiO}_{2}$ were determined, using a $(4 \times 4 \times 1)$ Monkhorst-Pack special k-points grid. Slab models containing 12, 14, 24, and 16 molecular units with areas of $14.5 \AA^{2}, 19.4 \AA^{2}, 35.9 \AA^{2}$, and $25.4 \AA^{2}$ for the (001), (101), (100), and (110) surfaces, respectively. It is worth noting that the (001) and (101) surfaces are O-terminated, while the (100) and (110) surfaces are $\mathrm{Ti}$ and O-terminated. Figure 4 depicts the slab representation for anatase $\mathrm{TiO}_{2}$. Each surface has been modeled using stoichiometric systems [119].

The $E_{\text {surf }}$ values and the Wulff construction of the ideal structure for anatase $\mathrm{TiO}_{2}$ in vacuum are shown in figure 5. It is possible to determine which structure should be present in anatase $\mathrm{TiO}_{2}$ by calculating the mathematical relationships

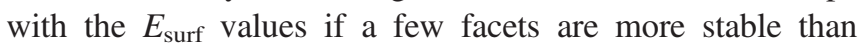
others. The Wulff constructions of those structures are also shown in figure 5.

Figure 5 can be used as a tool to verify the relative value of $E_{\text {surf }}$ that is necessary to obtain a particular morphology. Many research groups have studied anatase $\mathrm{TiO}_{2}$ with various shapes, such as single particle, nanosheet, cube, film, and flowerlike shapes [112-114].

Theoretical results reported by Barnard et al [121] point out the effect of water on the morphology of anatase and rutile $\mathrm{TiO}_{2}$. For anatase $\mathrm{TiO}_{2}$, the presence of water resulted in a variation in the size of the (001) facets. In another paper, Barnard and Curtiss [107] show the influence of acidic and alkaline conditions on the morphology of $\mathrm{TiO}_{2}$. The morphology shows a high occurrence of $(100) /(010)$ facets that predominantly occur in hydrogen-poor and oxygenated conditions. We obtained the surface energies of these shapedecreasing (100)/(010) facets.

Some experimental results reported recently by Pan et al show an elongated, truncated tetragonal bipyramid anatase with highly exposed (010) facets that was also synthesized by tuning the $\mathrm{Ti} / \mathrm{F}$ ratio [116]. This morphology was obtained by decreasing the $(100) /(010)$ surface energy of the initial Wulff construction on the map.

The cube shape is obtained by decreasing the (001) and (100)/(010) surface energy and was synthetized solvothermally by Zhao et al [111]. A scanning electron microscopy (SEM) image of anatase $\mathrm{TiO}_{2}$ particle synthesized by microwave-assisted hydrothermal method and its respective Wulff crystal is shown in figure 6. 


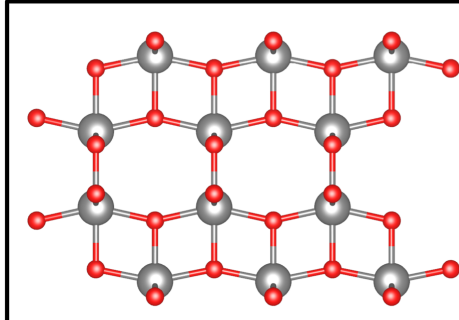

(001)

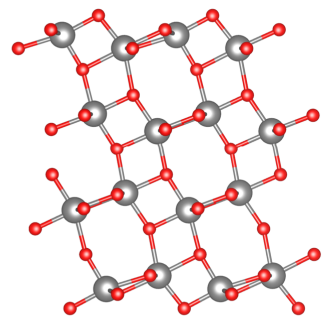

(101)

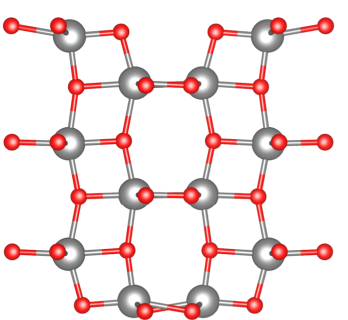

(100)

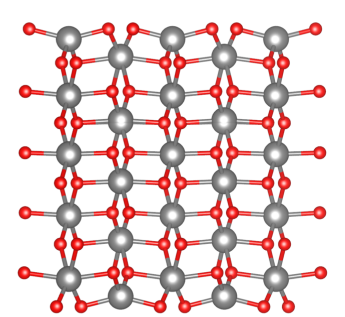

(110)

Figure 4. Surface models of (001), (101), (100), and (110) for anatase $\mathrm{TiO}_{2}$. The red and gray atoms represent $\mathrm{O}$ and $\mathrm{Ti}$ atoms, respectively.

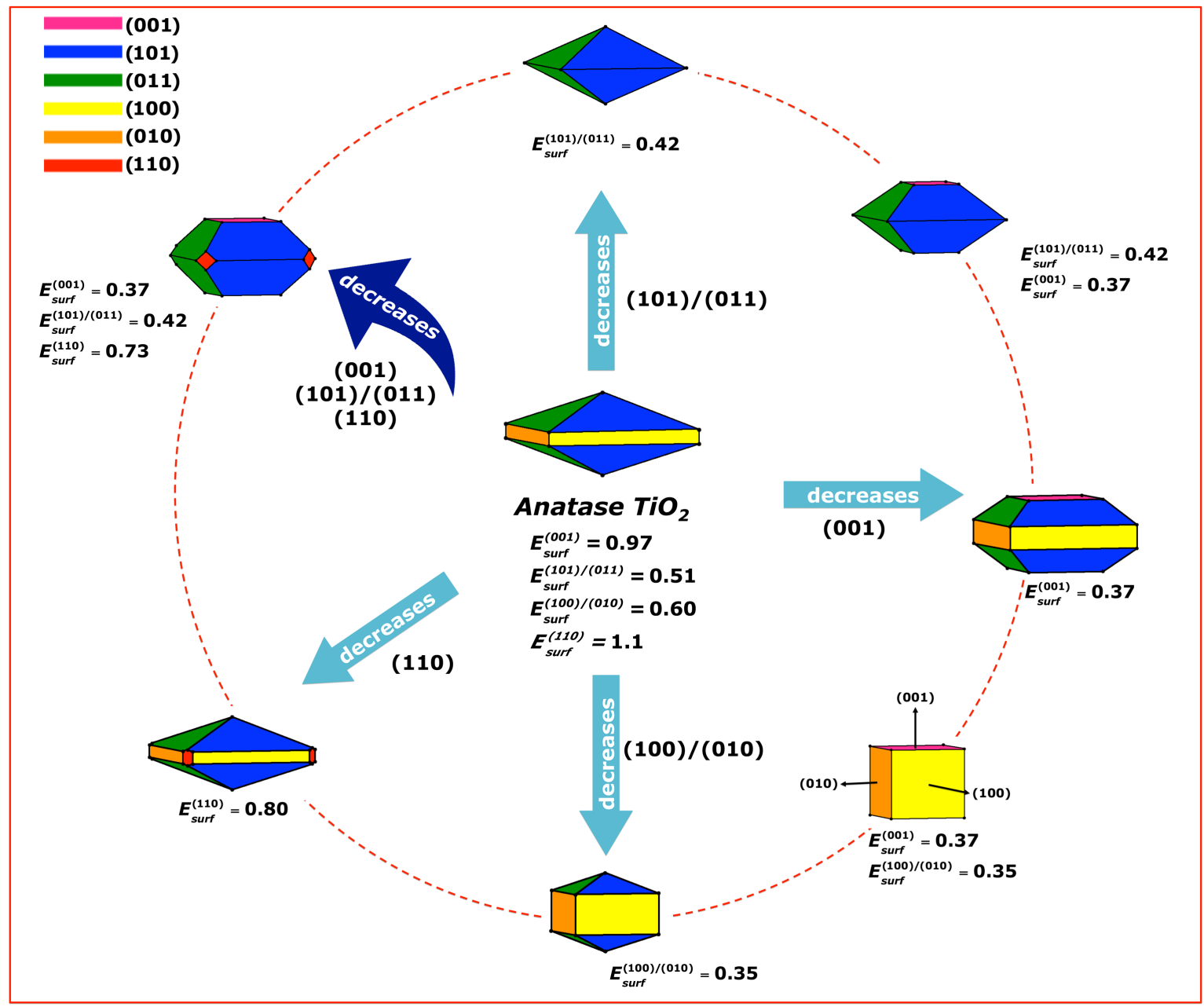

Figure 5. Crystallographic structure and map of morphologies of anatase $\mathrm{TiO}_{2}$ with crystal planes (001), (101), (011), (100), (010), and (110). Surface energy is in joule per-square meter. Theoretical (experimental) [120] lattice parameters, $a=3.806 \AA(3.78 \AA), c=9.722 \AA(9.51 \AA)$.

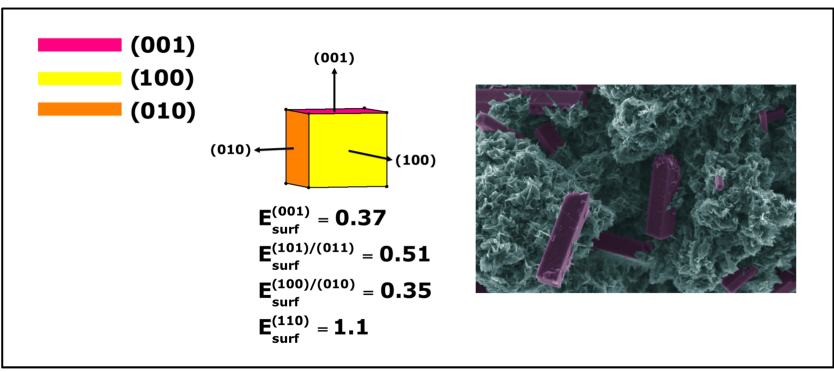

Figure 6. SEM image of cubic anatase $\mathrm{TiO}_{2}$ particle and its Wulff crystal representation. Surface energy is in joule per-square meter.
Even that the $\mathrm{TiO}_{2}$ particles showed in figure 6 have the same surfaces of the cubic morphology, they present a more elongated shape. This represents lower values of (100)/ (010) in relation of (001) surface. According to the Wulff theorem, the larger the difference is, the higher is this elongation.

Decreasing the (101)/(011) and (001) surface energy, we obtained the morphology synthesized by Yang et al that shows a breakthrough in synthesizing a truncated tetragonal bipyramid anatase $\mathrm{TiO}_{2}$ by fluorine capping, resulting in highly exposed (001) facets [42]. 
The octahedral structure was obtained by Amano et al using the hydrothermal reaction to synthetize a morphology with (101) facet [122]. The morphology with (001), (101), and (110) facets, as seen in the map, was obtained by Liu et al, which was synthesized using a modified hydrothermal technique in the presence of hydrogen peroxide and hydrofluoric acid solution [123].

\section{3. $\mathrm{BaZrO}_{3}$}

Barium zirconate $\left(\mathrm{BaZrO}_{3}\right)$ is a cubic perovskite at normal conditions, exhibiting a unique set of properties such as a high melting point, a small thermal expansion coefficient, small thermal conductivity, and excellent thermal and chemical stability [124]. These properties lead to the use of $\mathrm{BaZrO}_{3}$ in structural applications such as crucibles [125], thermal barrier coatings [126], components in wireless communication systems, the supersonic aircraft industry, and field emission displays, and it is also used as a green photocatalyst [127].

$E_{\text {surf }}$ of a plane is highly sensitive to the surface composition, impurities, and distortions/defects of a crystal. When an atom or foreign species becomes adsorbed on one surface or plane, it can decrease/increase the surface energy of that plane and may even stabilize/destabilize it with respect to other planes. Because of this adsorption, anisotropy is created in the surface energies, and this may be responsible for a change in the overall shape of the crystal with the original surface energy plane appearing/disappearing at the surface of the crystal. Traditionally, $\mathrm{KOH}$ (as a mineralizer) has often been used to provide an $\mathrm{OH}^{-}$environment in the hydrothermal crystallization process of $\mathrm{ABO}_{3}$ perovskite [128]. Morphology changes can also be accomplished by adding impurities/dopants to the system. The dopants act in a different way with respect to the changes in the energy order of the facets, according to the impurity species and their quantity [129].

Calculations for $\mathrm{BaZrO}_{3}$ bulk and surfaces were performed using Monkhorst-Pack special k-points grids $(3 \times 3 \times 1)$ to ensure geometrical and energetic convergence for the $\mathrm{BaZrO}_{3}$ structures considered in this work. The (001), (011), and (111) facets are modeled using stoichiometric systems. Slab models containing 6, 8, and 9 molecular units for (001), (011), and (111) surfaces, respectively, were considered, with areas of $18.1 \AA^{2}, 25.5 \AA^{2}$, and $31.3 \AA^{2}$, respectively. It is worth noting that the stoichiometric (001), (011), and (111) surfaces are $\mathrm{BaO}$ and $\mathrm{ZrO}_{2}$ terminated, $\mathrm{O}_{2}$ and $\mathrm{BaZrO}$-terminated, and $\mathrm{BaO}_{3}$ and $\mathrm{Zr}$-terminated, respectively, as illustrated in figure 7 .

Figure 8 presents the Wulff's crystal of the obtained $\mathrm{BaZrO}_{3}$ results. Figure 8 also shows all the possible morphologies that can be obtained by varying the ratio between the $E_{\text {surf }}$, taking into account the (001), (011), and (111) surfaces.

The variation in surface energy using the Wulff construction can be used as a powerful tool to evaluate the morphology of particles because of the difficulty in simulating all the details in a reaction system that interacts with the

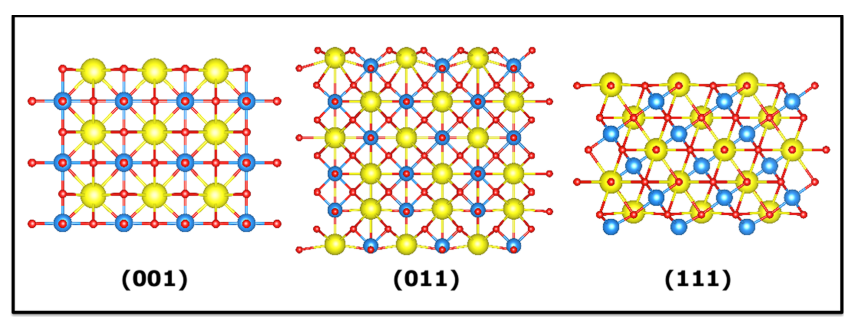

Figure 7. Surface models of (001), (011), and (111) surfaces for $\mathrm{BaZrO}_{3}$. The $\mathrm{Ba}, \mathrm{Zr}$, and $\mathrm{O}$ atoms are shown in yellow, blue, and red, respectively.

surface. A SEM image of a $\mathrm{BaZrO}_{3}$ particle synthesized by microwave-assisted hydrothermal method and its respective Wulff crystal is shown in figure 9.

Based on this result, it is possible to determine the relative energy values of each surface in order to obtain the experimental results. Besides the deca-octahedral morphology, Nakashime et al [131] reported cubic particles of $\mathrm{BaZrO}_{3}$ synthesized by a composite-hydroxide-mediated approach at low temperature. Moreira et al [132] also showed a coexistence of cubic and deca-octahedral $\mathrm{BaZrO}_{3}$ particles obtained by the microwave assisted hydrothermal method. These cases of cubic particles are in agreement with the ideal state (see figure 8 ).

\section{4. $\alpha-\mathrm{Ag}_{2} \mathrm{WO}_{4}$}

Transition metal tungstates are important inorganic materials that are studied and applied widely in many fields [133]. Among these, $\mathrm{Ag}_{2} \mathrm{WO}_{4}$ has attracted considerable attention because of their potential applications in fields as ionic conductivity [134], photocatalysis [135], sensors [136], microbial agents [137], photoluminescence properties [137, 138].

$\mathrm{Ag}_{2} \mathrm{WO}_{4}$ has three different crystallographic forms, $\alpha$-, $\beta$-, and $\gamma-\mathrm{Ag}_{2} \mathrm{WO}_{4}$, among which $\alpha-\mathrm{Ag}_{2} \mathrm{WO}_{4}$ is the thermodynamically stable phase that has been investigated [138]. To date, various methods of synthesis such as controlled precipitation [139], hydrothermal [140], and microwaveassisted method [89] have been reported in literature to obtain different $\mathrm{Ag}_{2} \mathrm{WO}_{4}$ nanostructures.

The (100), (010), (001), (110), (101), and (011) surfaces of $\alpha-\mathrm{Ag}_{2} \mathrm{WO}_{4}$ were modeled by unreconstructed slab models using a calculated equilibrium geometry and using a $(3 \times 3 \times 1)$ Monkhorst-Pack special k-points grid. Slab models containing $8,8,16,10,9$, and 16 molecular units for (100), (010), (001), (110), (101), and (011) surfaces, respectively, were considered, with areas of $68.6 \AA^{2}, 64.1 \AA^{2}$, $132.5 \AA^{2}, 93.9 \AA^{2}, 149.2 \AA^{2}$, and $147.2 \AA^{2}$, respectively. It is worth noting that the (010), (100), (101), and (011) surfaces are $\mathrm{O}$ and $\mathrm{Ag}$-terminated, while the (001) and (110) surfaces are $\mathrm{O}, \mathrm{W}$, and Ag-terminated. The slab representations are shown in figure 10 .

Figure 11 presents the Wulff's crystal of the optimized $\alpha$ $\mathrm{Ag}_{2} \mathrm{WO}_{4}$ and the different morphologies that would be obtained assuming different surface energy ratios. An analysis of figure 11 shows that the calculated energy surface values (ideal) generate a prism shape. The ratio of the energy surface 


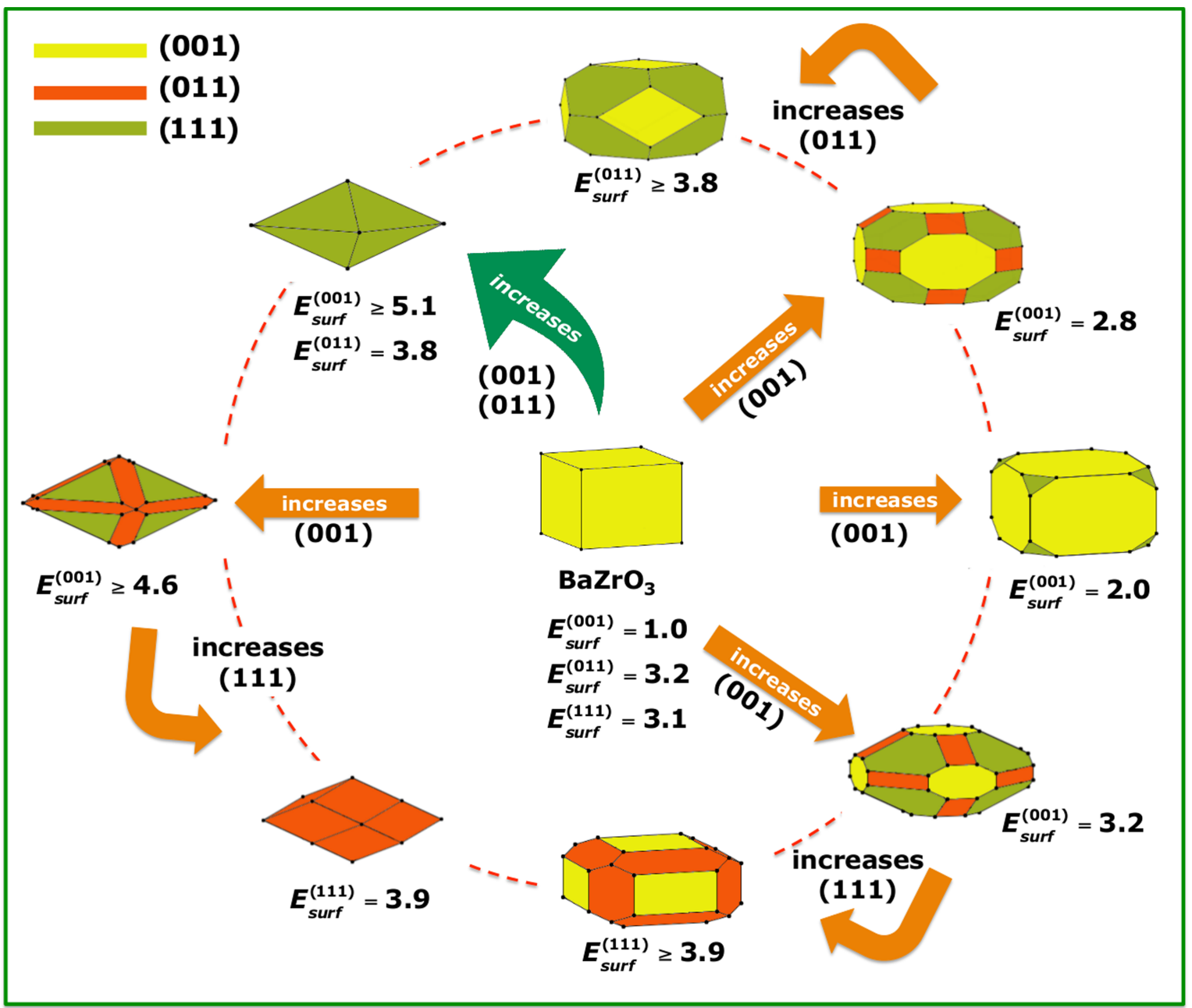

Figure 8. Crystallographic structure and map of morphologies of $\mathrm{BaZrO}_{3}$ with (001), (011), and (111) crystal planes. Surface energy is in joule per-square meter. Theoretical (experimental) [130] lattice parameter, $a=4.251 \AA$ ( $4.18 \AA$ ).

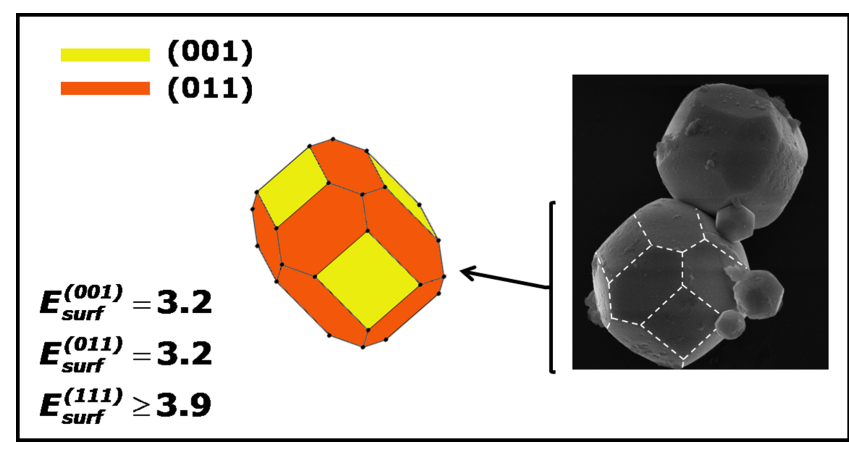

Figure 9. SEM image of deca-octahedral $\mathrm{BaZrO}_{3}$ crystals and its Wulff crystal representation. Surface energy is in joule per-square meter.

has been varied by increasing the value of the more stable surfaces (left in figure 11) and by decreasing the value of the less stable surfaces (right in figure 11).

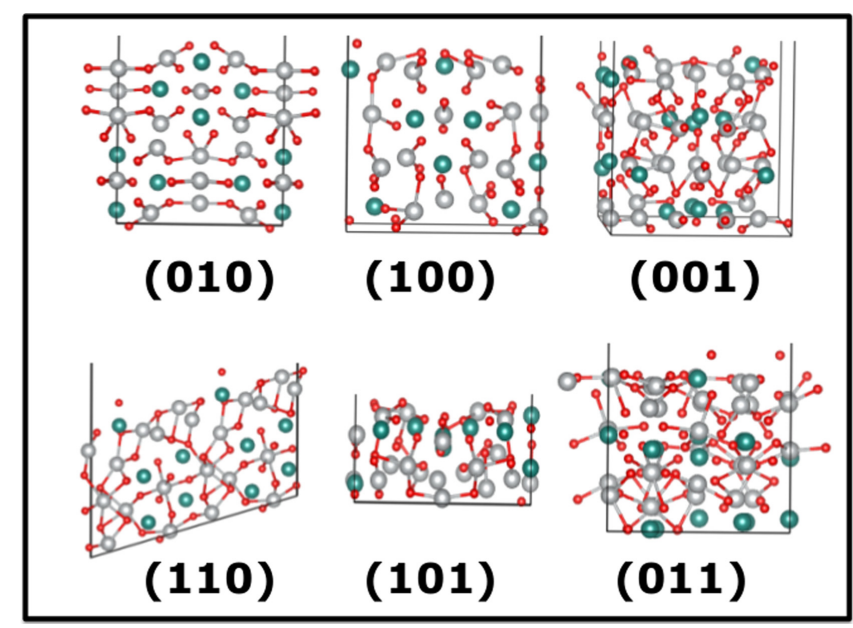

Figure 10. Surface models of (010), (100), (001), (110), (101), and (011) for $\alpha-\mathrm{Ag}_{2} \mathrm{WO}_{4}$. The $\mathrm{Ag}, \mathrm{W}$, and $\mathrm{O}$ atoms appear in gray, blue, and red, respectively. 


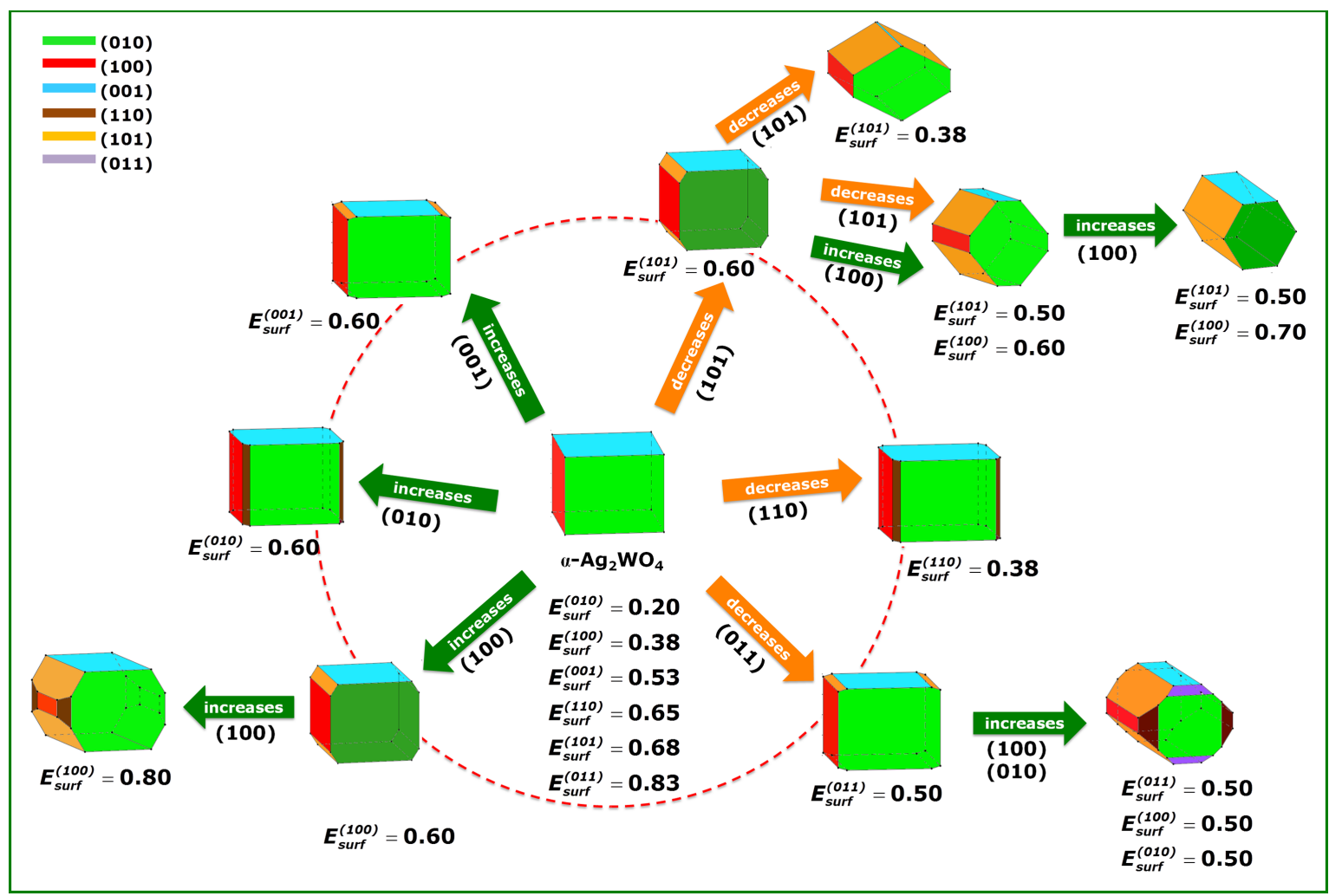

Figure 11. Crystallographic structure and map of morphologies of $\alpha-\mathrm{Ag}_{2} \mathrm{WO}_{4}$ with crystal planes (010), (100), (001), (110), (101), and (011). Surface energy is in joule per-square meter. Theoretical (experimental) [138] lattice parameters, $a=11.126 \AA(10.87 \AA), b=11.907 \AA$ $(12.01 \AA), c=5.761 \AA(5.89 \AA)$.

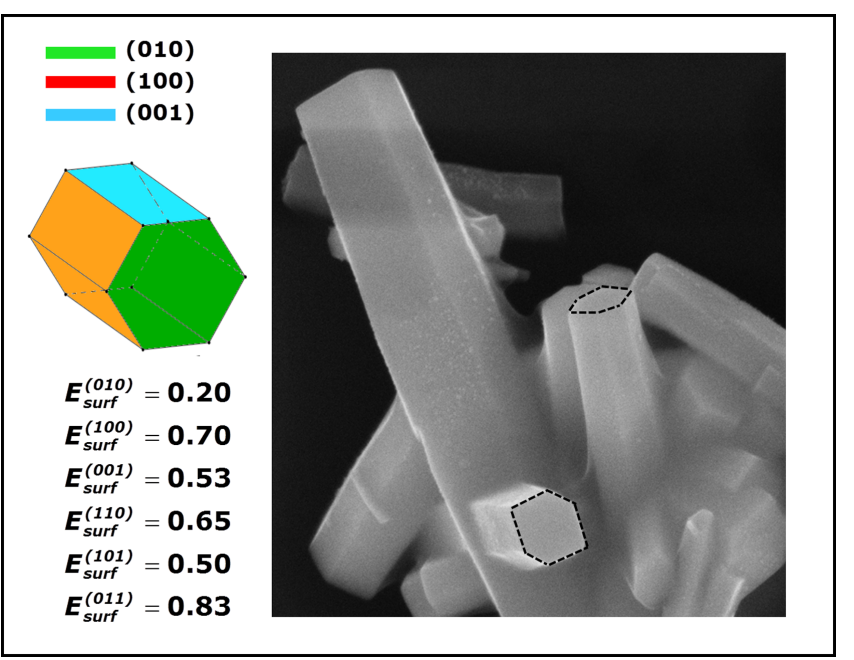

Figure 12. SEM image of $\alpha-\mathrm{Ag}_{2} \mathrm{WO}_{4}$ crystals and its Wulff crystal representation. Surface energy is in joule per·square meter.

A SEM image of $\alpha-\mathrm{Ag}_{2} \mathrm{WO}_{4}$ particle synthesized by microwave-assisted hydrothermal method and its respective Wulff crystal is shown in figure 12 . Besides this image, experimental results reported recently by Roca et al [60] show the same hexagonal rod-like shape under certain condition. Assuming the ideal structure as a starting-point, this morphology can be theoretically obtained increasing (101) and (100) surface energys to $0.5 \mathrm{~J} \mathrm{~m}^{-2}$ and $0.7 \mathrm{~J} \mathrm{~m}^{-2}$, respectively.
In summary, the mechanism of the morphology transformation of $\alpha-\mathrm{Ag}_{2} \mathrm{WO}_{4}$ crystals controlled by surface chemistry has been investigated experimentally and theoretically. The selection of surfaces to investigate was limited to low-index surfaces and surfaces with experimental observations were preferred.

\section{Conclusions}

The surface is a key component of a solid-state material, as many physical and chemical processes take place on surfaces. A series of materials that includes metal ( $\mathrm{Ag}$ ), simple (anatase $\left.\mathrm{TiO}_{2}\right)$, and complex $\left(\mathrm{BaZrO}_{3}\right.$ and $\left.\alpha-\mathrm{Ag}_{2} \mathrm{WO}_{4}\right)$ metal oxides with fascinating polyhedral morphologies such as octahedral, cuboctahedral, and truncated cubic structures were obtained. A controlled structural transformation between polyhedra is an essential goal of crystal engineering strategies. This framework is envisaged to provide guidelines for the synthesis of crystals of desired morphologies. The main conclusions can be summarized as follows: (i) We successfully demonstrate the morphological modulation of three-dimensional NCs. (ii) Based on the reverse Wulff theorem, we demonstrated that the structural transformations among different morphologies were easily controlled by the relative values of surface energies. (iii) By a proper adjustment of the ratio of the values for the surface energy of different facets, we are 
capable of finding a given morphology. This strategy will contribute to a better understanding of the control of morphological characteristics in nano- and micro-crystals. (iv) In the case of $\mathrm{Ag}$ and $\mathrm{BaZrO}_{3}$, it was applied a combination of experimental data, obtained from images taken by highresolution TEM and SEM, respectively, and the calculated surface energies for the three-dimensional Wulff construction. (v) This research will lay the foundations of not only unequivocal shape determination, but also an in-depth understanding of the observed features during the synthesis at the atomic level to help facilitate further breakthroughs in the control of crystal morphology.

Results presented here are an illustration of how firstprinciples calculations are capable of rationalizing the mechanisms stabilizing the morphology of micro- and nanoparticles at the atomic level. Using the present method, a 3D platform for the morphology modulation of materials with a complete array of accessible morphologies is obtained. We believe that our work has both predictive and explanatory capabilities, and it can be of help in the refinement of both research and technological developments to gain further understanding of how to achieve morphological control of complex three-dimensional crystals from metals to metal oxides.

\section{Acknowledgments}

The authors are grateful to FAPESP (2013/07296-2, 2012/ 14468-1, 2013/26671-9 and 2014/04350-9), CAPES (process A104/2013 and 99999.002998/2014-09), CNPq INCTMN 573636/2008-7, PrometeoII/2014/022 and ACOMP/2014/270 projects (Generalitat Valenciana), Ministerio de Economia y Competitividad (Spain), CTQ201236253-C03-02 and the Spanish Brazilian program (PHB20090065-PC) for financially supporting this research. We also acknowledge the Servei Informática, Universitat Jaume I for a generous allotment of computer time.

\section{References}

[1] Kong X Y, Ding Y, Yang R and Wang Z L 2004 Science 303 $1348-51$

[2] Huang S et al 2012 Dalton Trans. 41 5634-42

[3] Wang L and Li Y 2007 Chem. Mater. 19 727-34

[4] Zhai T et al 2009 Adv. Funct. Mater. 19 2423-30

[5] Flytzani-Stephanopoulos M and Schmidt L D 1979 Prog. Surf. Sci. 9 83-111

[6] Zhao X et al 2008 Funct. Mater. Lett. 01 167-72

[7] Sun C and Xue D 2014 Cryst. Growth Des. 14 2282-7

[8] Chen K, Sun C and Xue D 2015 Phys. Chem. Chem. Phys. 17 $732-50$

[9] Wulff G 1901 Z. Krystallogr. 34 449-530

[10] Herring C 1951 Phys. Rev. 82 87-93

[11] Roosen A R, McCormack R P and Carter W C 1998 Comput. Mater. Sci. 11 16-26

[12] Zucker R V, Chatain D, Dahmen U, Hagege S and Carter W C 2012 J. Mater. Sci. 47 8290-302

[13] Scopece D 2013 J. Appl. Crystallogr. 46 811-6
[14] Burda C, Chen X, Narayanan R and El-Sayed M A 2005 Chem. Rev. 105 1025-102

[15] Dahl J A, Maddux B L S and Hutchison J E 2007 Chem. Rev. $1072228-69$

[16] Lu A-H, Salabas E L and Schüth F 2007 Angew. Chem., Int. Ed. 46 1222-44

[17] Murray C B, Kagan C R and Bawendi M G 2000 Annu. Rev. Mater. Sci. 30 545-610

[18] Sun S, Murray C B, Weller D, Folks L and Moser A 2000 Science 287 1989-92

[19] Xia Y, Xiong Y, Lim B and Skrabalak S E 2009 Angew. Chem., Int. Ed. 48 60-103

[20] Kang Y, Pyo J B, Ye X, Gordon T R and Murray C B 2012 ACS Nano 6 5642-7

[21] Kang Y et al 2012 ACS Nano 7 645-53

[22] Peng X et al 2000 Nature 404 59-61

[23] Ye X et al 2010 Proc. Natl Acad. Sci. USA 107 22430-5

[24] Song Q, Ding Y, Wang Z L and Zhang Z J 2006 J. Phys. Chem. B 110 25547-50

[25] Somorjai G A 1981 Chemistry in Two Dimensions: Surfaces (Ithaca, NY: Cornell University Press)

[26] Zangwill A 1988 Physics at Surfaces (Cambridge: Cambridge University Press)

[27] Wang Z L 2000 J. Phys. Chem. B 104 1153-75

[28] Zhang G et al 2010 Appl. Surf. Sci. 257 302-5

[29] Di W, Willinger M-G, Ferreira R A S, Ren X, Lu S and Pinna N 2008 J. Phys. Chem. C 112 18815-20

[30] Yajuan S et al 2007 Nanotechnology 18275609

[31] Xia Y, Xiong Y, Lim B and Skrabalak S E 2009 Angew. Chem. 121 62-108

[32] Wen H et al 2008 J. Phys. Chem. C 112 15948-55

[33] Li Z, Lai X, Wang H, Mao D, Xing C and Wang D 2009 Nanotechnology 20

[34] Jian J K et al 2007 J. Cryst. Growth 303 427-32

[35] Wang H et al $2010 \mathrm{~J}$. Am. Chem. Soc. 132 15875-7

[36] Bratlie K M, Lee H, Komvopoulos K, Yang P and Somorjai G A 2007 Nano Lett. 7 3097-101

[37] Narayanan R and El-Sayed M A 2004 Nano Lett. 4 1343-8

[38] Ferrando R, Jellinek J and Johnston R L 2008 Chem. Rev. 108 845-910

[39] Buhro W E and Colvin V L 2003 Nat. Mater. 2 138-9

[40] Lim B et al 2009 Science 324 1302-5

[41] Xie X, Li Y, Liu Z-Q, Haruta M and Shen W 2009 Nature $458746-9$

[42] Yang H G et al 2008 Nature 453 638-41

[43] Joo J, Chow B Y, Prakash M, Boyden E S and Jacobson J M 2011 Nat. Mater. 10 596-601

[44] Jiang H B et al 2011 Angew. Chem., Int. Ed. 50 3764-8

[45] Dong J J et al 2011 ACS Appl. Mater. Interfaces 3 4388-95

[46] Kiatkittipong K, Scott J and Amal R 2011 ACS Appl. Mater. Interfaces 3 3988-96

[47] Maiyalagan T, Chemelewski K R and Manthiram A 2013 ACS Catal. 4 421-5

[48] Duan J, Chen S, Dai S and Qiao S Z 2014 Adv. Funct. Mater. 24 2072-8

[49] Yang W et al 2013 Electrochem. Commun. 28 13-6

[50] De Angelis F, Di Valentin C, Fantacci S, Vittadini A and Selloni A 2014 Chem. Rev. 114 9708-53

[51] Mudunkotuwa I A and Grassian V H 2011 J. Environ. Monit. 13 1135-44

[52] Karim A, Fosse S and Persson K A 2013 Phys. Rev. B 87 075322

[53] Kanaki E, Gohr S, Mueller C and Paulus B 2015 Surf. Sci. 632 158-63

[54] Barmparis G D, Lodziana Z, Lopez N and Remediakis I N 2015 Beilstein J. Nanotechnology 6 361-8

[55] Beltrán A, Andrés J, Longo E and Leite E R 2003 Appl. Phys. Lett. 83 635-7 
[56] Leite E R, Giraldi T R, Pontes F M, Longo E, Beltrán A and Andrés J 2003 Appl. Phys. Lett. 83 1566-8

[57] Stroppa D G et al 2014 Phys. Chem. Chem. Phys. 16 1089-94

[58] Bomio M R D et al 2013 J. Phys. Chem. C 117 21382-95

[59] Longo V M et al 2011 J. Phys. Chem. C 115 20113-9

[60] Roca R A et al 2015 Catalysis Sci. Technol. 5 4091-107

[61] Roca R A and Leite E R 2013 J. Am. Ceram. Soc. 96 96-102

[62] Kresse G and Furthmuller J 1996 Comput. Mater. Sci. 6 $15-50$

[63] Kresse G and Hafner J 1994 Phys. Rev. B 49 14251-69

[64] Perdew J P, Burke K and Ernzerhof M 1996 Phys. Rev. Lett. 77 3865-8

[65] Kresse G and Joubert D 1999 Phys. Rev. B 59 1758-75

[66] Heid R, Pintschovius L, Reichardt W and Bohnen K P 2000 Phys. Rev. B 61 12059-62

[67] McBride J R, Hass K C and Weber W H 1991 Phys. Rev. B 44 5016-28

[68] Baetzold R C and Yang H 2003 J. Phys. Chem. B 107 14357-64

[69] Barnard A S and Zapol P 2004 J. Chem. Phys. 121 4276-83

[70] Chizallet C et al 2008 J. Phys. Chem. C 112 16629-37

[71] Zasada F et al 2011 J. Phys. Chem. C 115 6423-32

[72] Vittadini A, Casarin M and Selloni A 2009 J. Phys. Chem. C 113 18973-7

[73] Rogal J, Reuter K and Scheffler M 2004 Phys. Rev. B 69 075421

[74] Lodziana Z, Topsoe N-Y and Norskov J K 2004 Nat. Mater. 3 289-93

[75] Selçuk S and Selloni A 2013 J. Phys. Chem. C 117 6358-62

[76] Wang Y et al 2014 Phys. Rev. B 90045304

[77] Rusanov A I 2005 Surf. Sci. Rep. 58 111-239

[78] Seyed-Razavi A, Snook I K and Barnard A S 2010 J. Mater. Chem. 20 416-21

[79] Jiang Z-Y, Kuang Q, Xie Z-X and Zheng L-S 2010 Adv. Funct. Mater. 20 3634-45

[80] Lee K, Kim M and Kim H 2010 J. Mater. Chem. 20 3791-8

[81] Chernov A 1963 Sov. Phys. Crystallogr. 7 728-30

[82] Sun Q, Yerino C D, Leung B, Han J and Coltrin M E 2011 J. Appl. Phys. 110053517

[83] Romanyuk K, Cherepanov V and Voigtländer B 2007 Phys. Rev. Lett. 99126103

[84] Gross E, LiuJack H-C, Toste F D and Somorjai G A 2012 Nat. Chem. 4 947-52

[85] Heveling J 2012 J. Chem. Educ. 89 1530-6

[86] Rycenga M et al 2011 Chem. Rev. 111 3669-712

[87] Zhang J, Li S, Wu J, Schatz G C and Mirkin C A 2009 Angew. Chem., Int. Ed. 48 7787-91

[88] Xia X, Zeng J, Zhang Q, Moran C H and Xia Y 2012 J. Phys. Chem. C $11621647-56$

[89] Longo E et al 2013 Sci. Rep. 31676

[90] Andrés J et al 2014 Sci. Rep. 55391

[91] Woehl T J, Park C, Evans J E, Arslan I, Ristenpart W D and Browning N D 2013 Nano Lett. 14 373-8

[92] Li D, Nielsen M H, Lee J R I, Frandsen C, Banfield J F and De Yoreo J J 2012 Science 336 1014-8

[93] Batson P E, Reyes-Coronado A, Barrera R G, Rivacoba A, Echenique P M and Aizpurua J 2011 Nano Lett. 11 3388-93

[94] Elechiguerra J L, Reyes-Gasga J and Yacaman M J 2006 J. Mater. Chem. 16 3906-19

[95] Rashkeev S N, Lupini A R, Overbury S H, Pennycook S J and Pantelides S T 2007 Phys. Rev. B 76035438

[96] Arumugam S K, Sastry T P, Sreedhar B and Mandal A B 2007 J. Biomed. Mater. Res. A 80A 391-8

[97] Yu A K, Kudrinskiy A A, Olenin A Y and Lisichkin G V 2008 Russ. Chem. Rev. 77233
[98] González A L, Noguez C, Beránek J and Barnard A S 2014 J. Phys. Chem. C 118 9128-36

[99] Sau T K and Rogach A L 2012 Complex-Shaped Metal Nanoparticles: Bottom-Up Syntheses and Applications (Weinheim, Germany: Wiley-VCH Verlag)

[100] Barnard A S 2014 Nanoscale 6 9983-90

[101] Wang Y, He J, Liu C, Chong W H and Chen H 2014 Angew. Chem., Int. Ed. 53 2-32

[102] Spreadborough J and Christian J W 1959 J. Sci. Instrum. 36 $116-8$

[103] Chen D and Caruso R A 2013 Adv. Funct. Mater. 23 1356-74

[104] Liu L and Chen X 2014 Chem. Rev. 114 9890-918

[105] Bourikas K, Kordulis C and Lycourghiotis A 2014 Chem. Rev. 114 9754-823

[106] Diebold U 2003 Surf. Sci. Rep. 48 53-229

[107] Barnard A S and Curtiss L A 2005 Nano Lett. 5 1261-6

[108] Yang H G and Zeng H C 2004 J. Phys. Chem. B 108 3492-5

[109] Gong X-Q and Selloni A 2005 J. Phys. Chem. B 109 19560-2

[110] Wen C Z, Zhou J Z, Jiang H B, Hu Q H, Qiao S Z and Yang H G 2011 Chem. Commun. 47 4400-2

[111] Zhao X et al 2011 Adv. Funct. Mater. 21 3554-63

[112] Wang Y, Zhang H, Han Y, Liu P, Yao X and Zhao H 2011 Chem. Commun. 47 2829-31

[113] Dinh C-T, Nguyen T-D, Kleitz F and Do T-O 2009 ACS Nano 3 3737-43

[114] Wen C Z, Hu Q H, Guo Y N, Gong X Q, Qiao S Z and Yang H G 2011 Chem. Commun. 47 6138-40

[115] Xiang Q, Yu J, Wang W and Jaroniec M 2011 Chem. Commun. 47 6906-8

[116] Pan J, Liu G, Lu G Q and Cheng H-M 2011 Angew. Chem., Int. Ed. $502133-7$

[117] D’Arienzo M et al 2011 J. Am. Chem. Soc. 133 17652-61

[118] Tachikawa T, Yamashita S and Majima T 2011 J. Am. Chem. Soc. 133 7197-204

[119] Bandura A V, Evarestov R A and Kuruch D D 2010 Surf. Sci. 604 1591-7

[120] Moura K F et al 2014 J. Solid State Chem. 210 171-7

[121] Barnard A S, Zapol P and Curtiss L A 2005 J. Chem. Theory Comput. 1 107-16

[122] Amano F, Yasumoto T, Prieto-Mahaney O-O, Uchida S, Shibayama T and Ohtani B 2009 Chem. Commun. 2311-3

[123] Liu M et al 2010 Chem. Commun. 46 1664-6

[124] Kumar H P et al 2008 J. Alloys Compd. 458 528-31

[125] Dierickx D, Houben I, Lapin J, Delannay F and Van Der Biest O 1996 J. Mater. Sci. Lett. 15 1573-6

[126] Pan L, Li L and Chen Y 2013 J. Sol-Gel Sci. Technol. 66 330-6

[127] Yuan Y et al 2008 Int. J. Hydrogen Energy 33 5941-6

[128] Wang D et al 2002 Solid State Ion. 151 329-33

[129] Alfredsson M et al 2007 Surf. Sci. 601 4793-800

[130] Moreira M L, Andres J, Varela J A and Longo E 2009 Cryst. Growth Des. 9 833-9

[131] Nakashima K, Fujii I and Wada S 2011 J. Ceram. Soc. Japan $119532-4$

[132] Moreira M L et al 2011 Scr. Mater. 64 118-21

[133] Yu S H, Liu B, Mo M S, Huang J H, Liu X M and Qian Y T 2003 Adv. Funct. Mater. 13 639-47

[134] Suthanthiraraj S A S and S 2012 Chem. Sci. Trans. 1 13-22

[135] Tang J and Ye J 2005 J. Mater. Chem. 15 4246-51

[136] da Silva L F et al 2014 Nanoscale 6 4058-62

[137] Longo V M et al 2014 J. Phys. Chem. A 118 5769-78

[138] Cavalcante L S et al 2012 Inorg. Chem. 51 10675-87

[139] Wang X, Li S, Yu H and Yu J 2011 J. Mol. Catal. A: Chem. 334 52-9

[140] Liu X et al 2013 Mater. Lett. 91 129-32 


\section{QUERY FORM}

JOURNAL: Nanotechnology

Author: J Andrés et al

TitLE: Effects of surface stability on the morphological transformation of metals and metal oxides as investigated by first-principles calculations

ARTICLE ID: nano519199

The layout of this article has not yet been finalized. Therefore this proof may contain columns that are not fully balanced/ matched or overlapping text in inline equations; these issues will be resolved once the final corrections have been incorporated.

SQ1

Please be aware that the colour figures in this article will only appear in colour in the online version. If you require colour in the printed journal and have not previously arranged it, please contact the Production Editor now.

\section{Page 10}

Q1

Please check the details for any journal references that do not have a link as they may contain some incorrect information.

\section{Page 10}

Q2

Please provide the page range or article number in reference [33].

Page 11

Q3

Please provide the volume number in reference [122].

\section{Page 11}

Q4

Please provide the surname for the author [S] in reference [134]. 